\title{
Communication and action predictability: two complementary strategies for successful cooperation
}

Mateusz Woźniak $^{1,2 *}$, Guenther Knoblich ${ }^{1}$

${ }^{1}$ Social Mind and Body Group, Department of Cognitive Science, Central European

University, Vienna, Austria

${ }^{2}$ Cognition and Philosophy Lab, Department of Philosophy, Monash University, Melbourne, Australia

* Corresponding author

E-mail: mgwozniak@gmail.com

Data: https://osf.io/3eqaj/

Word count: 9102 


\section{Abstract}

Making one's actions predictable and communicating what one intends to do are two strategies to achieve interpersonal coordination. It is less clear whether the two strategies of achieving coordination are mutually exclusive or whether they can be used in parallel. Here, we asked how the availability of communication channels affects the use of making one's actions predictable. In three experiments, we investigated how people reach joint decisions if they are not allowed to communicate at all (Experiment 1), allowed minimal reciprocal communication (Experiment 2), or allowed to use the full range of conventional communication (Experiment 3). We found that when participants were not allowed to communicate coordination was achieved by increasing action predictability. When conventional communication was allowed there were no attempts to increase action predictability. In the minimal reciprocal communication condition, successful pairs both increased action predictability and established a communication system. Overall, the present study demonstrates that people are able to flexibly adapt to coordination challenges during joint decision making and that communication reduces behavioral constraints on joint action coordination.

Keywords: communication, predictability, cooperation, decision making, coordination 


\section{INTRODUCTION}

The ability to cooperate and coordinate is the basis not only for being able to achieve common goals in pairs and small groups, but also lays foundation for large societies (Raihani, 2021; Rand \& Nowak, 2013; Sachs, Mueller, Wilcox, \& Bull, 2004). It also underpins the extraordinary evolutionary success of the human race. Successful cooperation is not necessarily easy and straightforward. It requires coordination of decisions and actions between two or more people, often under uncertain and difficult circumstances. What are the cognitive mechanisms that allow humans to overcome these obstacles and make successful cooperation possible?

Thomas Schelling (Schelling, 1960) initiated this discussion in the 1960s by proposing one strategy: doing what is predictable for others. He argued that if people need to coordinate their decisions, but cannot communicate with each other, they can increase the chance of successful coordination by making their decisions predictable. A classic example is a situation where two individuals agreed to meet at a certain time, but haven't decided on the place of the meeting. They leave their houses and go into the city. They will succeed if they both head towards the same location (regardless of where it is) and lose time and effort if they head to two different locations and consequently fail to meet. Under such circumstances people often choose a salient well-known landmark, such as Karl's Church in Vienna, or a similar outstanding spot. This strategy is known as using "focal points", i.e. choosing salient options that we believe that might be chosen by default by other people (Mehta, Starmer, \& Sugden, 1994; Sugden, 1995). A focal point might be also a place where the involved individuals have met repeatedly before - in this case it would be established by a history of repeated predictable behaviors (Crawford \& Haller, 1990).

Similar strategies exploiting other forms of predictability can be used in other situations (Michael, McEllin, \& Felber, 2020; Michael \& Pacherie, 2015) reflecting a general rule: coordination can be facilitated by strategic use of predictability of others' behavior. Moreover, 
recent research provides rich evidence that predictability is used to facilitate cooperation and coordination across a wide range of contexts and situations going beyond decision making. When performing joint actions people reduce the variability of their movements to smooth coordination with each other (Sabu, Curioni, Vesper, Sebanz, \& Knoblich, 2020; Sacheli, Tidoni, Pavone, Aglioti, \& Candidi, 2013; Vesper, Schmitz, Safra, Sebanz, \& Knoblich, 2016; Vesper, van der Wel, Knoblich, \& Sebanz, 2011). For example, in a recent study (Glover \& Dixon, 2017) found that timing of motor action was much more predictable in cooperative than in competitive context. These studies show that making one's action more predictable is one of the basic mechanisms facilitating cooperation between individuals.

The situation when two people need to blindly meet at the same spot might seem abstract in the contemporary world in which $2 / 3$ of the world population possesses cell phones that allow anyone to simply call and discuss where to meet. Cell phones are a recent invention, but throughout the history of mankind people utilized many ways to communicate with each other to achieve common goals. Communication represents the second strategy supporting interpersonal coordination (Tylén, Weed, Wallentin, Roepstorff, \& Frith, 2010). Communication is the main facilitator to achieve successful cooperation, when people can use a conventional language. However, communication can also be used to foster cooperation and coordination when a shared conventional language is not available. In such cases people communicate through the use of sensorimotor communicative behaviors (McEllin, Knoblich, \& Sebanz, 2018; Pezzulo et al., 2019; Vesper, Schmitz, \& Knoblich, 2017) or develop new communication systems from scratch (Fay, Ellison, \& Garrod, 2014; Galantucci, 2017; Galantucci \& Garrod, 2011; Misyak, Noguchi, \& Chater, 2016; T. Scott-Phillips, 2014; Tamariz, 2017).

It is well established that both action predictability and communication can serve as strategies facilitating successful cooperation between individuals. However, what is less 
explored is whether both strategies are used in an exclusive manner or whether both strategies can be combined and whether combining both strategies happens by default or only if necessary. There is evidence showing that people adapt the way that they use language to make interaction smoother (Fusaroli et al., 2012; Lelonkiewicz \& Gambi, 2020; Lindsay, Gambi, \& Rabagliati, 2019), showing that predictability can be used to fine-tune communication. However, an empirical investigation of how the availability of communication modulates motivation to make one's behavior predictable is missing. In the current study, we conducted three experiments to systematically vary the degree to which participants could communicate with each other while participating in a coordination game.

\section{EXPERIMENT 1}

The goal of the first experiment was to investigate whether people spontaneously use behavioral predictability to boost coordination with another person during a multi-trial joint decision-making task. Pairs of participants played a cooperative version of the Rock-PaperScissors (RPS) game. They were instructed to cooperate to choose the same object (rock, paper, or scissors). There was no possibility to communicate verbally or non-verbally. The only information that was available to participants was whether they had successfully made a joint decision or not. To increase coordination difficulty, in each trial one of the choices was randomly made unavailable. The unavailable choice was independently randomly selected for each participant. This made it impossible for the participants to coordinate by reliably choosing the same option.

To establish a baseline for coordination success, the same pairs of participants also performed a modified competitive version of the standard RPS game where one participant was instructed to choose the same option as the other person, while the other participants was 
instructed to choose the "winning" option from RPS. In all other respects the baseline condition was the same as the cooperative condition.

We hypothesized that in the absence of any possibility to communicate participants would make their behavior more predictable in the cooperative condition than in the baseline condition in which participants were competing against each other. We also expected that the increased use of a strategy to make one's own choices predictable would lead participants to achieve better (and above-chance-level) performance compared to the baseline.

\subsection{METHODS}

\subsubsection{Participants}

Forty-two volunteers participated in the study comprising 21 dyads. One dyad was excluded from the analysis, because one of the participants reported during the post-experiment briefing that he misunderstood the task instructions. The mean age of the remaining 40 participants was 27.45 years $(\mathrm{SD}=5.1)$. Eighteen participants were females. All participants were right-handed, except for two left-handed, and one ambidextrous. The participants represented diverse national and linguistic backgrounds. All of them were fluent in English, while none was a native English speaker. Two participants reported that they did not know the Rock-Paper-Scissors game prior to the experiment. All participants gave informed consent in a written form, and the study was approved by the Ethical Research Committee of Central European University, in accordance with the standards from the Declaration of Helsinki (applies to all experiments).

\subsubsection{Procedure, Stimuli, and Apparatus}

Pairs of participants' played a modified Rock-Paper-Scissors (RPS) game. In each trial each participant independently chose one of three objects: rock, paper, or scissors. Before the 
experiment started participants practiced the competitive RPS game to familiarize them with the setup. During practice participants were allowed to ask questions until they fully understood the task and setup. After the practice ended, participants were no longer allowed to verbally communicate, until the end of the experiment. After practice participants performed a cooperative version and a baseline (competitive) version of the task (order of versions was counterbalanced across pairs of participants) - instructions about the rules of the cooperative task were provided only immediately before the cooperative task started.

In the cooperative task participants were instructed to make the same choice as their partner on each trial. For every trial in which both participants made the same choice (e.g. both chose "paper") they both received 2 points, and for every trial in which they made different choices they both received 0 points. In the baseline version of the task they were playing a modified rock-paper-scissors game. Participant A's task was the same as in the cooperative task: to make the same choice as participant B (receiving 2 points if successful and otherwise 0 points). Participant B was instructed to choose a winning option against participant A according to the standard rules of the RPS game, i.e.: rock beats scissors, paper beats rock, and scissors beat paper. Participant B received 2 points for the winning option and 0 points for the nonwinning option. There were three possible outcomes of each round in the baseline task: participant A wins (when both made the same choice), participant B wins (when B made a winning choice over A), and both lose (when B makes a "losing" choice against A). It is important to emphasize that the individual goal of participant A was exactly the same in the cooperative and the baseline task: to make the same choice as participant B. Only the context of the choice differed for participant A because participant received a competitive instruction in the baseline task.

Figure 1 illustrates the experimental setup. At the beginning of the experiment participants were seated at two sides of a table. A computer screen was placed in the lying 
position on the surface of a table, and a wooden structure was positioned at the center of the table. The wooden structure served two functions. First, it prevented the participants from seeing each other and their partner's side of the screen. Second, there were two button boxes placed on top of the wooden construction, which participants used to provide their choices, hence they will be called the "response boxes". Moreover, additional button boxes were placed right in front of each participant. They were used for the participants to proceed to the next trial after giving a response, and they will be called the "auxiliary boxes".

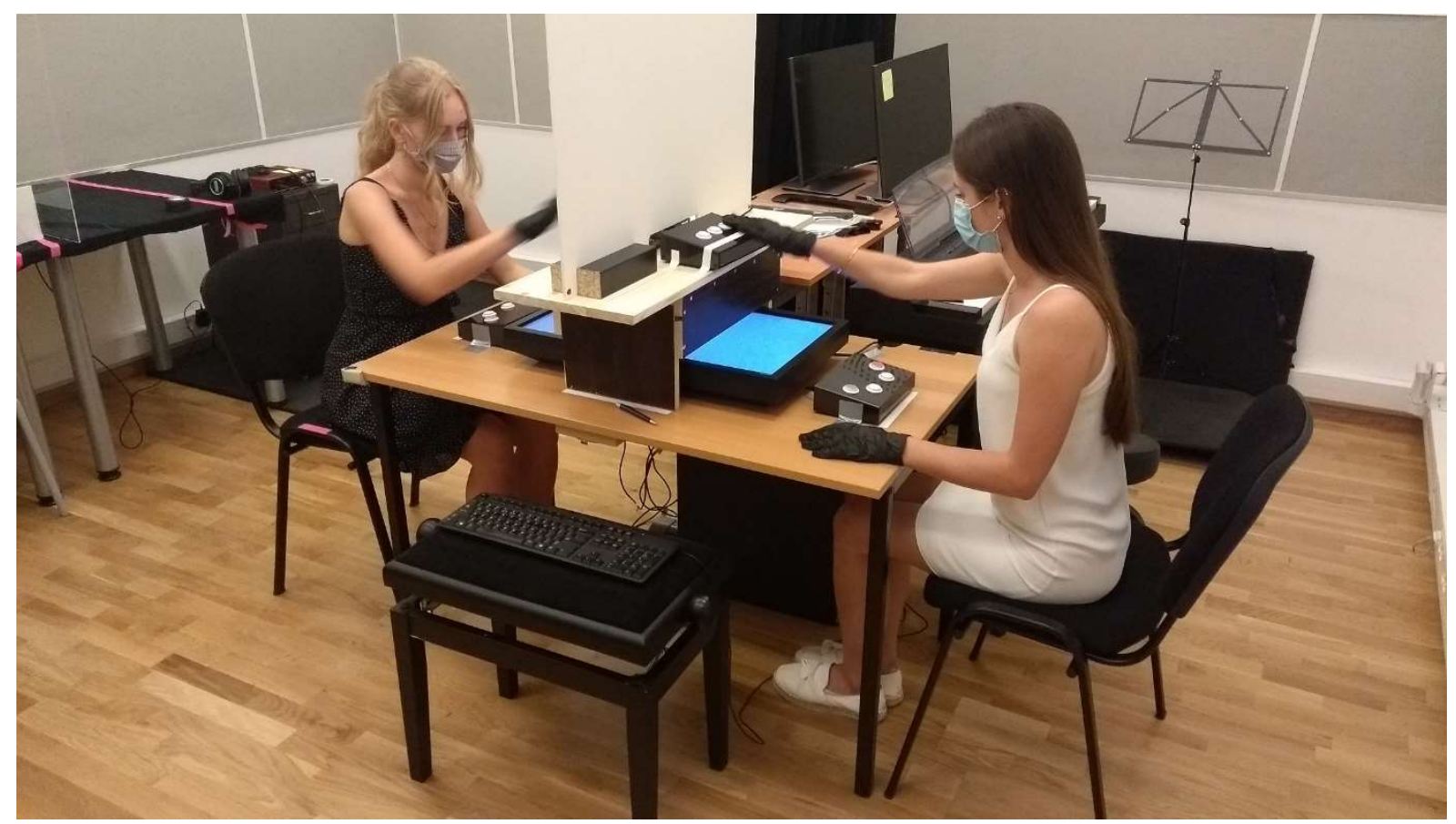

Figure 1. The experimental setup.

Figure 2 illustrates the time course of an individual trial for a single participant. Each trial started after both participants pressed and held "start-trial" buttons (pink buttons in figure 1) from their auxiliary boxes. Each participant saw a fixation cross on the screen for $1000 \mathrm{~ms}$. Afterwards, they saw three symbols: one on the left, one in the center, and one on the right. These symbols provided information about which button of the response box represented which choice. For example, if the symbol on the right represented a rock, then pressing the response 
button on the right indicated "rock" choice in a given trial. After seeing the symbols participants decided which option to choose, and pressed a button representing their choice. Importantly, in each trial one of the options was "blocked". This was indicated by an "X" drawn over one of the options. Participants were discouraged from choosing the "blocked" option because it automatically resulted in 0 points for both participants in a given trial.

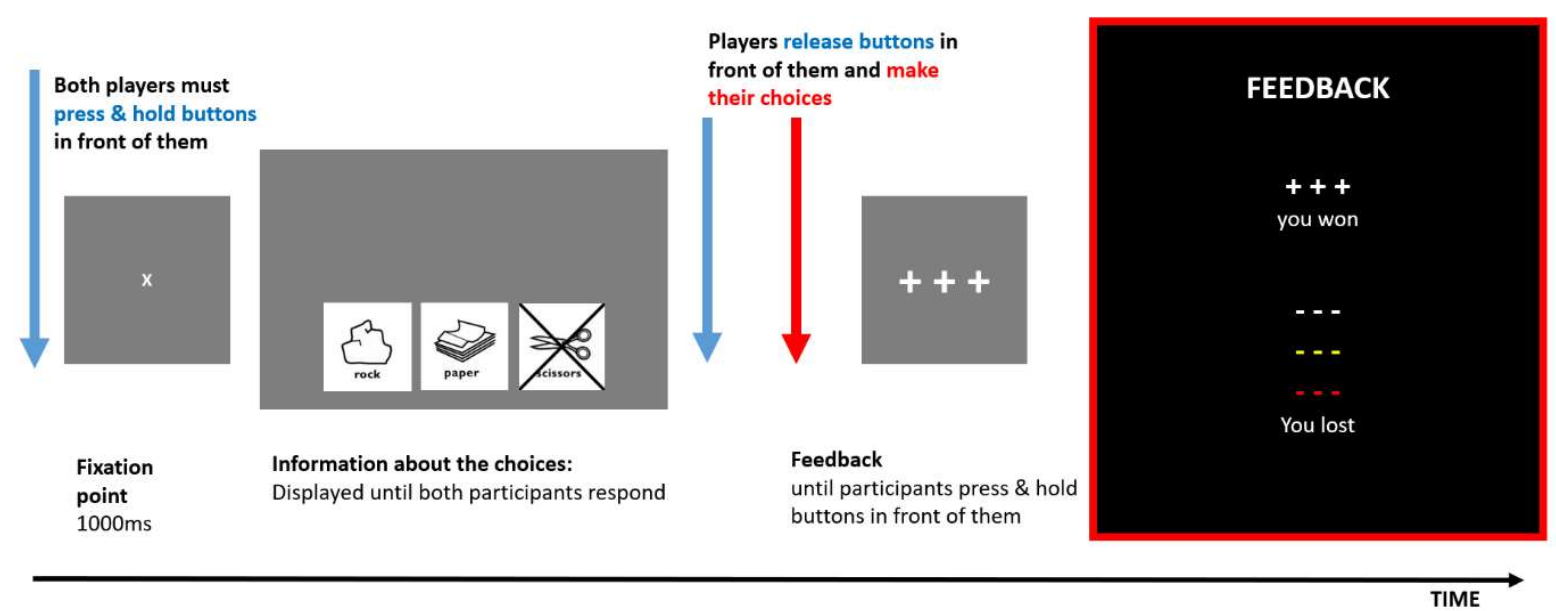

Figure 2. An example of the time course of a single trial for an individual participant, and symbols used to indicate different types of feedback.

The assignment of choices to buttons of a response box (six possible combinations), as well as the blocked options changed randomly for each participants from trial to trial - each option was blocked equal amount of times in each experimental block. Thus in most cases the assignment of choices to buttons and which option was blocked was different for each participant of a dyad. Importantly, the information about available choices for Participant A provided was unrelated to the available choices for Participant B and vice versa.

Participants were encouraged to try to make their choices at the same time as their partner. If their key presses did not occur within a $1000 \mathrm{~ms}$ time window both did not receive points for the trial. After both participants made their choices by pressing respective buttons at 
the response boxes, each received feedback displayed on the screen: " +++ " in white indicated success, “_ _ _" in white indicated failure, “_ _ _" in red indicated that at least one of the participants made a mistake by either choosing a "blocked" option or by pressing a decision button more than once, and "- - -" in yellow indicated that the asynchrony between their choices was greater than 1000 ms. Feedback was presented on the screen until both participants pressed and held their fingers on the "start-trial" buttons of the auxiliary boxes. This initiated the next trial.

Each dyad performed the cooperative and the baseline task. The order of tasks was counterbalanced across dyads. For each task participants performed 12 blocks of 18 trials. The whole experiment lasted between 40 and 60 minutes. The experiment was programmed and run in Matlab R2017a with Psychophysics Toolbox (version 3.0.10). All text was presented in white on grey background.

\subsubsection{Research design and data analysis}

The experiment comprised a single-factor within-subject design comparing Task: cooperative vs. competitive. Moreover, the competitive condition included an additional nested factor of Role (Participant A vs. Participant B: between-subject factor). Participant A's task was to always choose the same option as Participant B, while Participant B had to choose the same option as Participant A in the cooperative task, but a "winning option" against Participant A in the competitive task, making the competitive task a version of the "matching pennies" game.

The dependent variables were: (1) Win rate: average percentage of wins in a block or a task, with chance level of $33 \%$ because there were three possible choices, (2) Choice entropy: entropy of choices made by a participant within a block (measured in bits) and calculated as:

$$
\text { Choice entropy }=-\sum_{i=1}^{3}\left(p_{i} \log _{2} \mathrm{p}_{i}\right)
$$


with $p_{\odot}$ being the probability that a certain symbol $\subset$ (rock, paper, scissors) was chosen by the participant in each block. Choice entropy reflects the predictability of choices made by a participant. For the present task, the maximum value of 1.585 bits reflects a situation in which a participant equally often chose rock, paper and scissors - meaning that the choices were random (chance level) and there was no preference for any choice over the others. A theoretical value of 0 bits would mean that a participant chose the same option (e.g., paper) in all trials of a given block corresponding to perfect predictability. However, because in the present task each option was blocked in $33 \%$ of the trials, the minimum value of choice entropy that participants could achieve was 0.918 bits, reflecting the most predictable pattern of responses (choosing one option $66 \%$ of the time, and a selected second option $33 \%$ of the time).

Data analysis was conducted using custom scripts written in Matlab R2017a and using JASP 0.9.0.1. Violations of the assumption of sphericity were estimated using Mauchly's W test and the Greenhouse-Geisser correction was applied whenever this assumption was violated.

All data and analysis scripts are available at the OSF depository under the following link: https://osf.io/3eqaj/

\subsection{RESULTS}

In the cooperative task $2.2 \%(S D=2.3 \%)$ trials were excluded because participants had made a mistake (pressing the button for the "blocked" option, pressing the same button twice etc.). This amounted on average to 4.75 mistakes per participant per task (range between 0 and 19 mistakes $)$. In the competitive task also $2.2 \%(S D=2.3 \%)$ of trials were excluded due to mistakes (the range of the number of mistakes per participant was also between 0 and 19).

In the cooperative task we conducted a one-way ANOVA to test whether participants were more likely to choose some options over another and we discovered that participants displayed a strong preference $\left(F(2,78)=8.02, p<0.001\right.$, partial $\left.\eta^{2}=0.17\right)$ : rock was chosen most 
frequently $(42.2 \%)$, while paper $(28.7 \%)$ and scissors $(28.2 \%)$ were chosen less often. In the competitive task we conducted a two-way ANOVA with factors Choice (rock vs. paper vs. scissors) and Role (Participant A vs. Participant B). Participants showed unequal preference for different choices as indicated by the main effect of Choice $(F(2,76)=7.18, p=0.001$, partial $\left.\eta^{2}=0.16\right)$ : on average they were choosing rock more often $(36.7 \%)$ than scissors $(32.3 \%)$ and scissors more often than paper (30.0\%). Neither Role $(F(1,38)=0.01, p=0.91)$ nor Role $\mathrm{x}$ Choice interaction $(F(2,76)=0.11, p=0.90)$ were significant. Additional results in reaction times and movement asynchronies for all experiments are reported in the supplementary materials (S1).

\subsubsection{Win rate}

A repeated-measures t-test revealed a significant effect of Task $(t(39)=6.09, p<0.001$, Cohen's d=0.96, $\mathrm{BF}_{10}=41914$ ) showing that participants obtained significantly more points in the cooperative than in the competitive task. The comparison between win rate of Participant A and B in the competitive task revealed no significant differences $(t(39)=0.76, p=0.46$, Cohen's $\left.\mathrm{d}=0.24, \mathrm{BF}_{10}=2.58\right)$.

In the second step we investigated the number of wins at the level of individual blocks with an additional two-way repeated-measures ANOVA $(2 \times 12)$ with factors: Task (competitive vs. cooperative) and Block (from block 1 to block 12). The results revealed a significant main effect of Task $\left(F(1,39)=37.16, p<0.001\right.$, partial $\left.\eta^{2}=0.49, \mathrm{BF}_{\text {inclusion }}=3.6 \mathrm{e} 13\right)$ whereas the main effect of block was not significant $(F(11,429)=1.25, p=0.27$ Greenhouse-Geisser corrected, partial $\left.\eta^{2}=0.03, \mathrm{BF}_{\text {inclusion }}=0.002\right)$. The interaction effect was not significant $(F(11,429)=1.61$, $p=0.14$, partial $\left.\eta^{2}=0.04, \mathrm{BF}_{\text {inclusion }}=5.3 \mathrm{e}-4\right)$. 

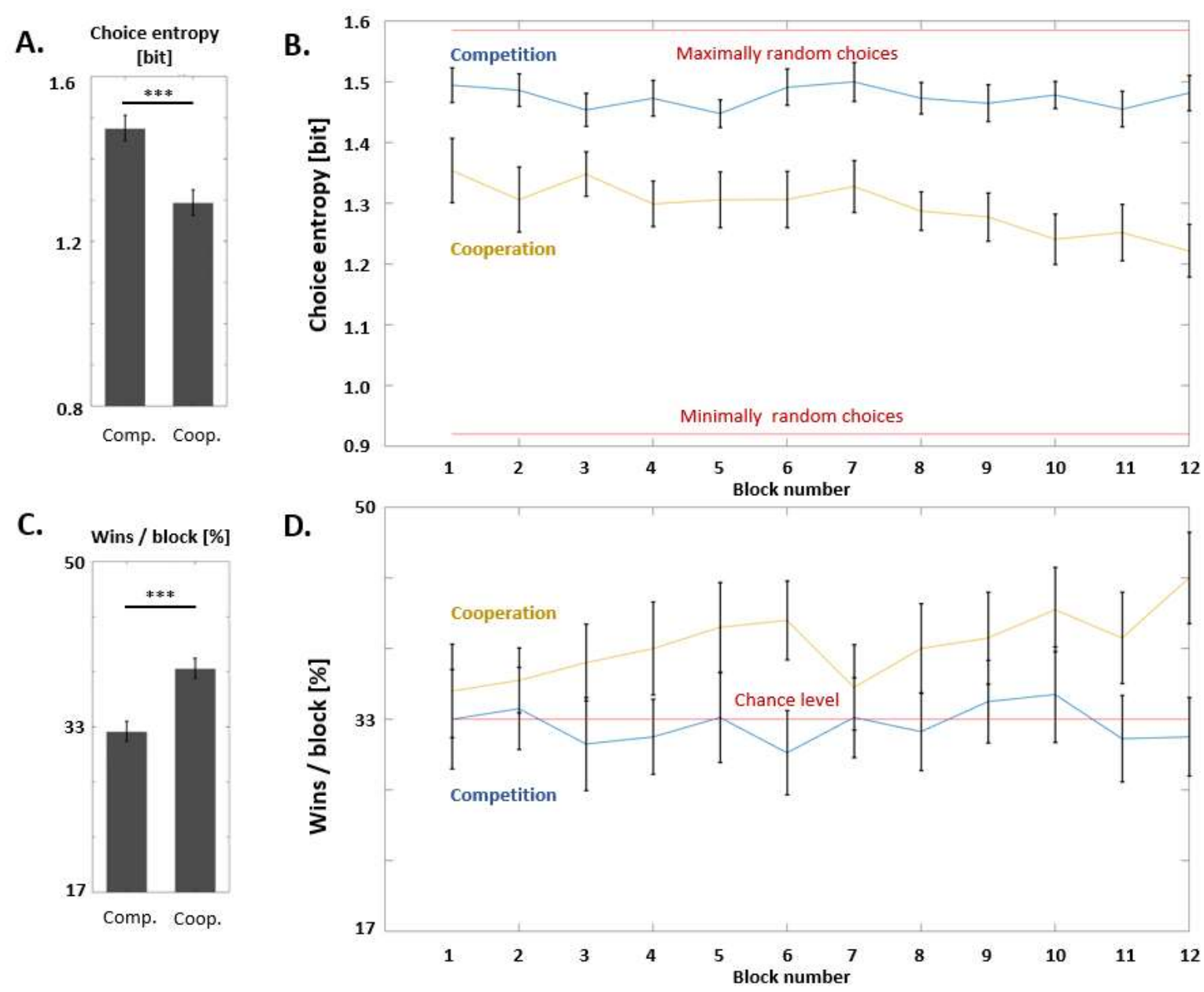

Fig 3. Results of Experiment 1. Bar graphs on the left illustrate the differences between competitive and cooperative tasks in average choice entropy (A) and win rate (C). Line graphs on the right show average choice entropy (B) and win rate (D) across individual blocks in competitive and cooperative task. Brackets indicate 95\% Cis.

\subsubsection{Choice entropy}

A repeated-measures t-test revealed a significant effect of Task $\left(t(39)=5.68 \_, p<0.001\right.$, Cohen's $\left.\mathrm{d}=0.90, \mathrm{BF}_{10}=12253\right)$ indicating that participants displayed smaller choice entropy (meaning: more predictable choices) in the cooperative than in the competitive task. The comparison between win rate of Participant A and B in the competitive task revealed no significant differences $\left(t(39)=0.70, p=0.46\right.$, Cohen's $\left.\mathrm{d}=0.22, \mathrm{BF}_{01}=2.66\right)$. 
In the next step we investigated choice entropy at the level of individual blocks with an additional two-way repeated-measures ANOVA $(2 \times 12)$ with the factor Task (competitive vs. cooperative) and Block (from block 1 to block 12). The results revealed that the main effects of Task $\left(F(1,39)=32.3, p<0.001\right.$, partial $\left.\eta^{2}=0.45, \mathrm{BF}_{\text {inclusion }}=\mathrm{inf}\right)$ and block $(F(11,429)=3.25$, $p=0.003$ Greenhouse-Geisser corrected, partial $\left.\eta^{2}=0.08, B F_{\text {inclusion }}=0.017\right)$ were significant. The interaction was not significant $(F(11,429)=2.0, p=0.078$ Greenhouse-Geisser corrected, partial $\left.\eta^{2}=0.05, \mathrm{BF}_{\text {inclusion }}=0.003\right)$.

\subsubsection{Correlations between variables}

In order to estimate the relationship between behavioral predictability and task success we calculated Spearman's rho correlations between choice entropy and number of wins independently for the competitive and cooperative task. In both they were negatively correlated: in the competitive task the correlation was $\operatorname{rho}(38)=-0.384(p=0.014)$, and in the cooperative task it was rho(38) $=-0.584(p<0.001)$.

\subsubsection{Cooperation strategies}

In the present task only one cooperation strategy could ensure success: Participants needed to converge on choosing one specific option whenever possible, and to choose a second specific option if the first option was blocked. For example, the participants in a pair needed to always choose "rock" if it was not blocked, to always choose "paper if "rock" was blocked, and to never choose "scissors". Out of 40 participants 30 reported that at some point they were using a variant of this strategy, although they often failed to be consistent in choosing a second option when the first option was blocked.

Several other strategies were also reported by the participants. Six of them reported that they were always choosing an option that would win in a standard Rock-Paper-Scissors game if such an option was available. Three participants reported that they were trying to follow the conventional order of the sequence of gestures: first rock, then paper, then scissors. One 
participant was always pressing a middle button, and if this option was blocked then the right button. Finally, six participants reported that they did not use any strategy at all.

A complementary perspective on strategies used by participants during the cooperative task can be seen when inspecting the dynamics of choice entropy and win rate across blocks for each individual pair of participants (see Figure 4). Some pairs managed to successfully coordinate their strategies, or at least improve over time (e.g. P2, P5, P9, P11, P16, P18, P19). This was usually accompanied by an increase in win rate. In some cases, one of the participants adopted the first step of the optimal strategy (always choosing the same option, unless it was blocked), while the other did not. In such situations the former either maintained high predictability across most of the experiment (P7, P8, P13) or became discouraged and reverted to random choice (P3, P14, P15). In both situations the pair's win rate was usually close to chance-level performance. Finally, many pairs showed complex dynamics of choice entropy and win rate, most likely reflecting the process of unsuccessful adjustments of participants to each other.
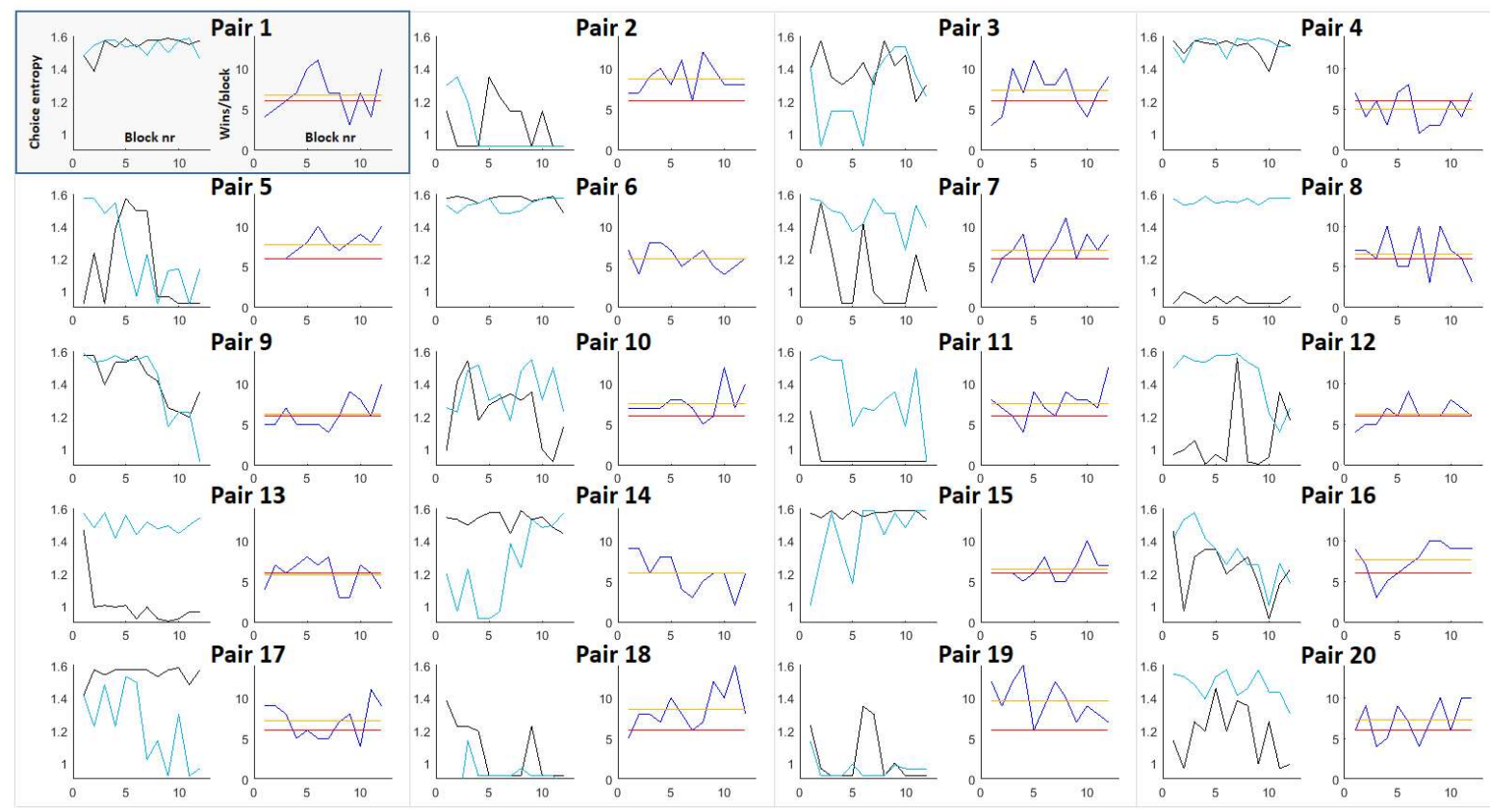

Figure 4. Choice entropy (graphs with black and teal lines) and numbers of wins (graphs with blue, red, and orange lines) in the cooperative task across blocks for each of 20 dyads that 
participated in Experiment 1. Teal lines represent Participants A and black lines - Participants B. Blue lines correspond to the number of wins by each dyad in cooperative trials in each block, while the orange lines reflect their average number of wins. The red lines correspond to the amount of wins expected by chance if both participants responded randomly (six wins per block).

\subsection{DISCUSSION}

The first experiment demonstrated that individual participants were behaving in a more predictable way in the cooperative task than in the competitive task, as indicated by the significant difference in choice entropy. This difference in action predictability enabled pairs to achieve a higher win rate in the cooperative context. Most frequently (75\%) participants increased their predictability by consistently choosing the same option if available, which is one important ingredient of a strategy to improve joint decisions in the present task. The second important ingredient is to converge on choosing the same option and on a second option to be used if the preferred options was blocked. As shown by the individual results, in the absence of the possibility to communicate, only few pairs were successful with agreeing on the same prioritization of options.

We did not find any differences in the behavior of Participants A and Participants B. It means that our results do not reflect the specifics of the task (whether a participant has to choose the same option as one's partner or a "beating” option), but instead that they are fully driven by whether the context was competitive or cooperative. Moreover, the same task goal of Participants A ("choose the same option as the other person") led to drastically different behavior depending on the context of the interaction, showing that our pattern of results is not modulated by a proximal goal, but reflects a general change of strategy to act in the task. 
In the competitive task entropy of choices was much higher than in the cooperative task. It was close to theoretically possible maximum, although it did not reach that value. This finding can be explained by the fact that maximum entropy can be obtained in a situation when participants equally often choose each option within a block, i.e. six times "rock", six times "paper", and six times "scissors". This, however, would introduce a higher-order predictability into their behavior. Therefore, the pattern of results showing choice entropy slightly lower than the theoretical maximum is what one should expect if participants tried to respond fully randomly, but also is expected if participants engaged in some form of strategically non-random behavior.

\section{EXPERIMENT 2}

The goal of Experiment 2 was to investigate whether participants can simultaneously use two coordination strategies, predictability and communication, when both are available but none of them alone guarantees joint success. We used the same task as in Experiment 1 but introduced a small change in the cooperative condition: Each participant was allowed to send one 1-bit signal to their partner before they made their choices. We did not allow the participants to communicate in the baseline condition.

Moreover, in Experiment 2 we abandoned the distinction between Participant A and Participant B in competitive context, because in Experiment 1 we did not find any evidence that this manipulation affects the results (neither directly nor through interaction). Therefore, in Experiment 2 the competitive task was the standard RPS game in which both players were guided by the same rules, to choose the winning option against their opponent.

\subsection{METHODS}

\subsubsection{Participants}


Forty volunteers comprising 20 dyads participated in the study. The mean age of the participants was 26.58 years $(\mathrm{SD}=3.5)$. Twenty-one participants were females. All participants were right-handed, except for five left-handed, and one ambidextrous. The participants represented diverse national and linguistic backgrounds and none of them was a native English speaker.

\subsubsection{Procedure}

The procedure of Experiment 2 was identical to Experiment 1 with two exceptions. First, and most importantly, in each trial of the cooperative task participants were given a chance to send a signal to their partner by pressing a "send-signal" button on the auxiliary box (grey buttons in Figure 1) after they lifted their finger from the "start-trial" button, and before they made their choice using the response box. To send the signal they used the same hand that they used to operate the other buttons. After a participant pressed a "send-signal" button, two green rectangles were displayed on the screen until the end of the trial - each of them visible to only one participant. A participant who pressed a button (sender) saw a green rectangle below the RPS images, and the other participant (receiver) saw it above the RPS images. It was possible that both participants sent a signal, that only one of them did, or that none of them did.

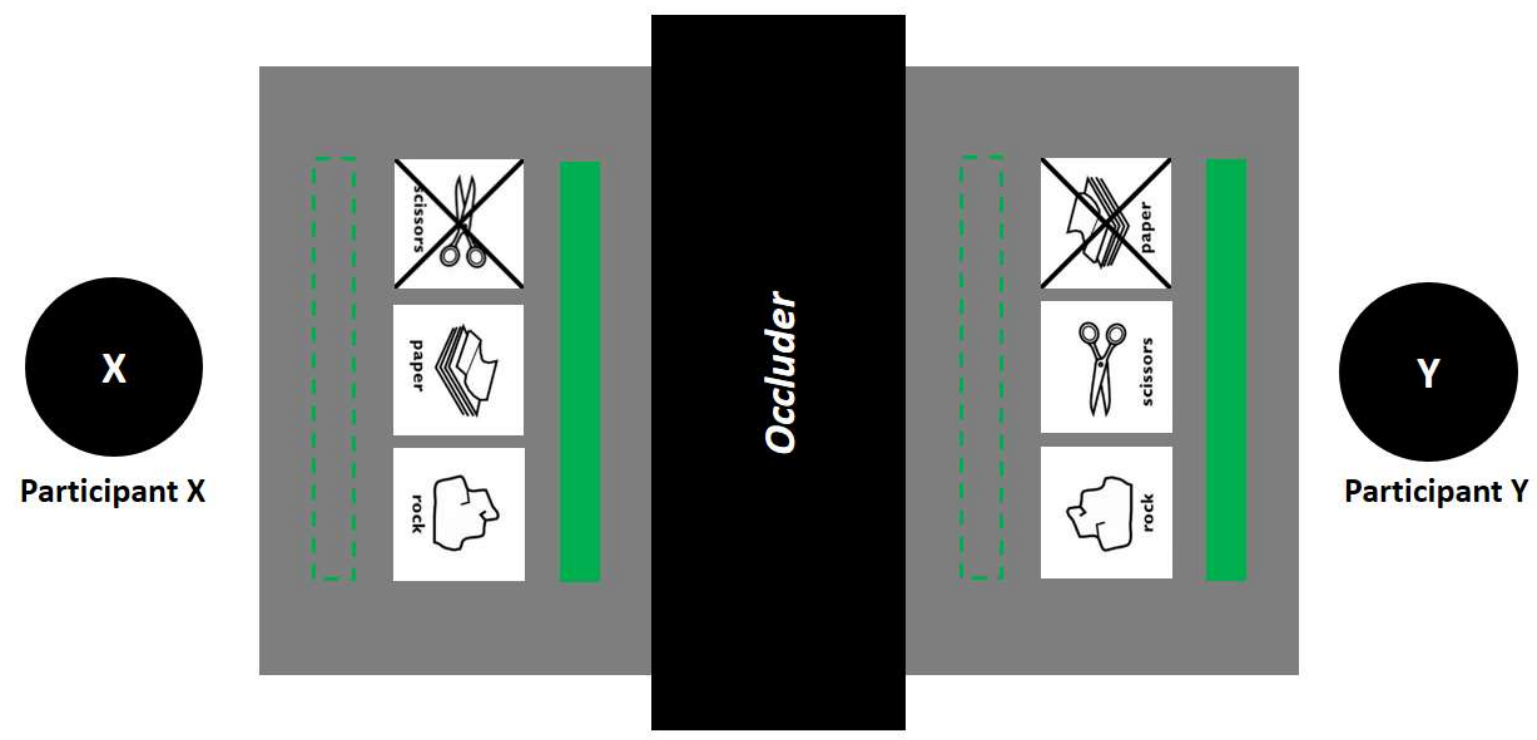


Fig 5. An illustration of how participants could send signals to each other in Experiment 2. In this example participant $\mathrm{Y}$ sent a signal to participant $\mathrm{X}$, as indicated by a solid green rectangle below the RPS pictures on Y's side of the screen, and a solid green rectangle above the RPS pictures on X's side of the screen. If participant $X$ decides to also send a signal, then solid green rectangles will also appear in the places indicated by the dashed contours of rectangles (dashed contours were not displayed during the experiment).

The second difference was that in the competitive context both participants had the same task, which was the standard Rock-Paper-Scissors task: to choose the "winning" option against the other participant. If participant chose a "winning" option, then they received 2 points for a given trial. If participant chose a "losing" option or the same option then they received 0 points.

\subsubsection{Research design and data analysis}

The experiment comprised a single-factor repeated-measures design comparing competitive vs. cooperative task. Signal informativeness was added as an additional dependent variable. Signal informativeness is a measure of average informational content (in bits) conveyed by each participant within each block of the cooperative task and was calculated using the following formula:

$$
\text { Signal informativeness }=-\sum_{i=0}^{1}\left(p_{i} \log _{2} \mathrm{p}_{i}\right)
$$

With $p_{1}$ being probability of sending a signal by a participant during a given block, and $p_{0}$ reflecting probability of not sending the signal. (so, $p_{1}+p_{0}=1$ ). Signal informativeness can take values between 0 and 1 bit, with 0 bits reflecting situations in which a participant either did not use signaling at all during a block, or was signaling in every trial (making the signal 
non-informative), and 1 bit reflecting a situation in which a participant was signaling during half of the trials in a given block.

Preregistration for Experiment 2 is available at: https://aspredicted.org/2j84g.pdf Full results are available in the supplementary materials S2.

\subsection{RESULTS}

In the competitive task $2.2 \%$ trials $(S D=1.8 \%)$ were excluded due to participants' mistakes (pressing a "blocked" option, pressing the same button twice etc.). This amounted to on average 4.7 mistakes per participant (the range in both tasks was between 0 and 13 mistakes). In the cooperative task $4.1 \%$ trials $(S D=3.3 \%)$ were excluded due to participants' mistakes (pressing a "blocked" option, pressing the same button twice etc.). This amounted to on average 8.95 mistakes per participant (the range in both tasks was between 1 and 29 mistakes). The difference in the number of mistakes was significantly larger for the cooperative than the competitive task $(\mathrm{t}(39)=4.93, \mathrm{p}<0.001)$.

Participants did not show unequal preferences for different choices: rock, paper or scissors, neither in the competitive task $\left(F(2,78)=0.61, p=0.55\right.$, partial $\left.\eta^{2}=0.02\right)$, nor in the cooperative task $\left(F(2,78)=0.25, p=0.78\right.$, partial $\left.\eta^{2}<0.01\right)$.

\subsubsection{Win rate}

A repeated-measures t-test revealed a significant effect of Task $(t(39)=4.75, p<0.001$, Cohen's $d=0.75, B F_{10}=783$ ) showing that participants obtained significantly more points in the cooperative than in the competitive task.

At the second step, we investigated the number of wins at the level of individual blocks. We conducted an additional two-way repeated-measures ANOVA $(2 \times 12)$ with the factors Task (competitive vs. cooperative) and Block (from block 1 to block 12). The results revealed a 
significant main effect of Task $\left(F(1,39)=22.53, p<0.001\right.$, partial $\left.\eta^{2}=0.37, \mathrm{BF}_{\text {inclusion }}=6.0 \mathrm{e} 15\right)$ a significant main effect of Block $(F(11,429)=6.99, p<0.001$ Greenhouse-Geisser corrected, partial $\left.\eta^{2}=0.15, \mathrm{BF}_{\text {inclusion }}=7.3 \mathrm{e} 12\right)$, and a significant interaction $(F(11,429)=9.32, p<0.001$, partial $\eta^{2}=0.19, B F_{\text {inclusion }}=3.7 \mathrm{e} 8$ ).
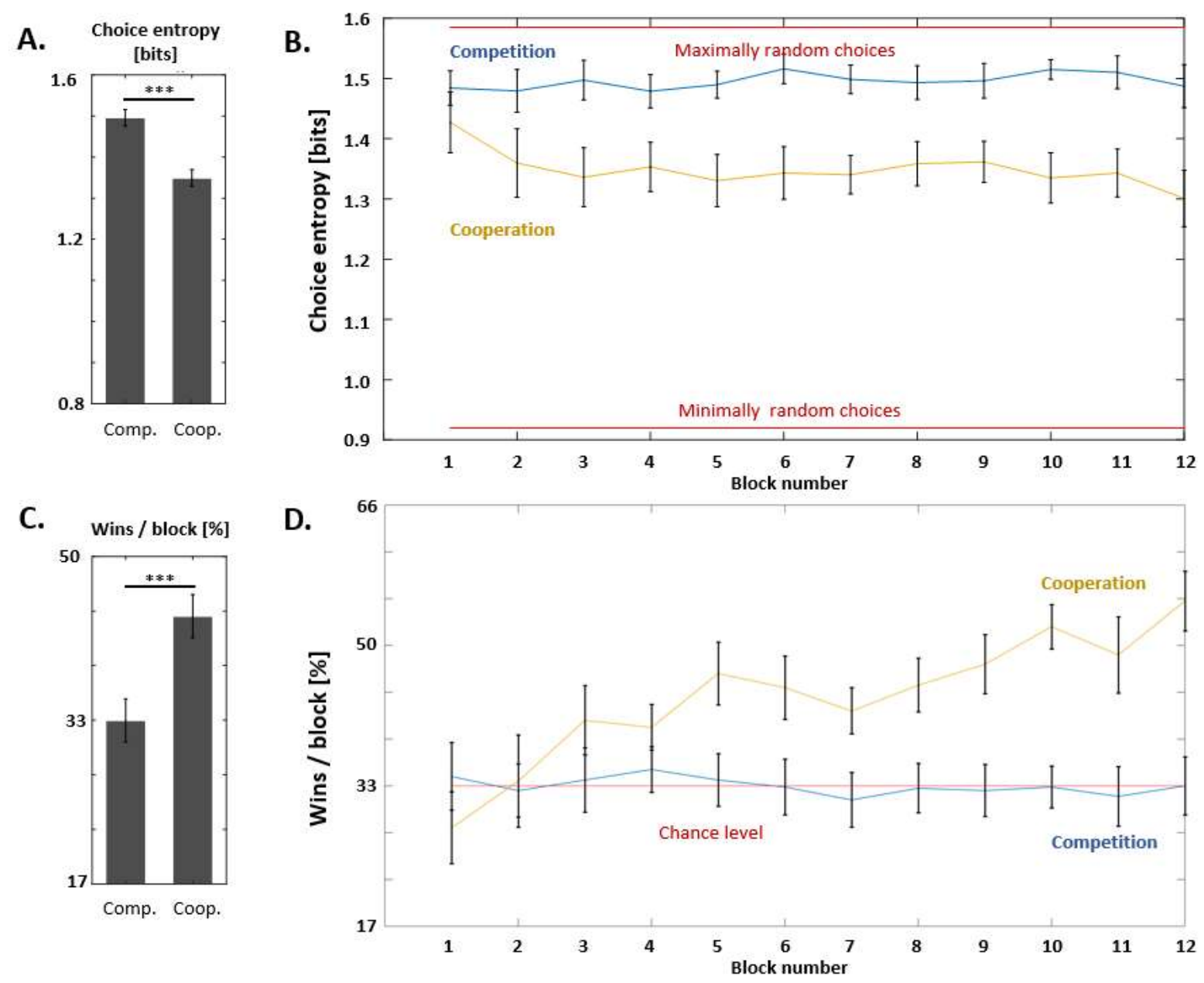

Fig 6. Results of Experiment 2. Bar graphs on the left illustrate the differences between competitive and cooperative task in average choice entropy (A) and win rate (C). Line graphs on the right show average choice entropy (B) and win rate (D) across individual blocks in competitive and cooperative context. Brackets indicate 95\% CIs.

\subsubsection{Choice entropy}


A repeated-measures t-test revealed a significant main effect of Task $(t(39)=7.09$, $p<0.001$, Cohen's $\mathrm{d}=1.12, \mathrm{BF}_{10}=8.19 \mathrm{e} 5$ ) indicating that participants displayed significantly smaller choice entropy in the cooperative than in the competitive context.

In the next step we investigated choice entropy at the level of individual blocks. We conducted an additional two-way repeated-measures ANOVA $(2 \times 12)$ with the factors Task (competitive vs. cooperative) and Block (from block 1 to block 12). The results revealed that the main effect of Task $\left(F(1,39)=50.28, p<0.001\right.$, partial $\eta^{2}=0.56$, BF $_{\text {inclusion }}=$ inf $)$ was significant. The main effect of Block $(F(11,429)=1.15, p=0.34$ Greenhouse-Geisser corrected, partial $\left.\eta^{2}=0.03, \quad B F_{\text {inclusion}}=5.4 \mathrm{e}-4\right)$ and the interaction effect were not significant $\left(F(11,429)=1.57, p=0.15\right.$ Greenhouse-Geisser corrected, partial $\left.\eta^{2}=0.04, \mathrm{BF}_{\text {inclusion }}=8.9 \mathrm{e}-5\right)$.

\subsubsection{Correlations between variables}

Similarly to Experiment 1, we estimated the relationships between our dependent variables. In the competitive task we did not find a significant correlation between choice entropy and win rate $(\operatorname{rho}(38)=-0.06, p=0.73)$, but we found a significant relationship in the cooperative task $(\operatorname{rho}(38)=-0.32, p=0.043)$ that was numerically smaller than in Experiment 1 . In the cooperative task we also investigated the relationship of signal informativeness with win rate, which was strongly positive $(\operatorname{rho}(38)=0.65, p<0.001)$. Finally and importantly, correlation between signal informativeness and choice entropy was strongly negative $(\operatorname{rho}(38)=-0.52$, $p<0.001$ ), meaning that participants who provided more informative signals were also making their behavior more predictable.

\subsubsection{Comparison of successful and unsuccessful pairs}

As the next step of the analysis, we performed an exploratory analysis in which we compared pairs that succeeded in cooperative task with pairs that did not. To differentiate between them we conducted $\mathrm{k}$-means clustering (with $\mathrm{k}=2$ ) based on the average number of wins across all blocks resulting in classification of all pairs into one of two groups. The first 
group involved pairs that did not succeed in the cooperative task. It consisted of 11 pairs and achieved on average 5.93 wins/block (range between 4.58 and 6.92). The second group consisted of 9 pairs and achieved on average 10.31 wins/block (range between 8.83 and 13.08). The difference in the average number of wins between these groups was significant $(t(18)=8.38$, $p<0.001$, Cohen's d=3.77, $\left.\mathrm{BF}_{10}=6.9 \mathrm{e}+4\right)$.

To get further insight into the differences between these groups we compared them based on the dependent variables. The choice entropy of participants belonging to the successful group was only marginally smaller $\left(\mathrm{t}(38)=1.91, p=0.064\right.$, Cohen's $\left.\mathrm{d}=0.61, \mathrm{BF}_{10}=1.28\right)$, but their average signal informativeness was significantly higher $(\mathrm{t}(38)=3.98, p<0.001$, Cohen's $\mathrm{d}=1.26$, $\mathrm{BF}_{10}=84.2$ ) compared to pairs from the unsuccessful group.

\subsubsection{Cooperation strategies}

In contrast to Experiment 1, in Experiment 2 there were multiple ways of succeeding in the cooperative task. Most of them required using the possibility to send signals between partners. The strategy from Experiment 1 to always choose one option whenever it was not blocked was still possible, but it was almost never successfully used, as indicated by the fact that successful pairs showed a rather high choice entropy, including in blocks in which they scored the highest points. Instead, all successful pairs, and most pairs in general, engaged in some form of communicative behavior. In Pair 13 one of the participants was using signaling at almost every individual trial, making signaling uninformative. The other participant attempted to provide informative signals during the first six blocks, but afterwards stopped signaling altogether. A similar dynamic happened in Pair 15, where one of the participants was not using signaling at all, while the other one started with frequent signaling, but abandoned this possibility after the first 4 blocks. Both pairs achieved a close-to-chance-level average number of wins. 
Pairs that succeeded in coordinating their choices reported using several strategies. The simplest one included one participant sending a signal indicating a specific choice (e.g., "I have a rock") and the other participant made a choice depending on this information. This one-way communication strategy did not fully utilize the communicative potential, but was enough to achieve above-chance performance. In our case it was used by Pair 1. All other successful pairs developed some versions of a two-way communication strategy. In the most typical strategy one of the participants was sending a signal to indicate her or his willingness to choose a certain option. For example, in Pair 11 one of the participants was sending a signal whenever she wanted to choose paper, while her partner was signaling whenever he wanted to choose scissors, and they made their decisions based on the configuration of who sent and who did not send a signal. Similarly, in Pair 16 one participant was signaling paper and the other one rock, in Pair 8: rock and scissors, in Pair 6 both participants were signaling if they had paper. A slightly different strategy was developed by Pair 14 where one participant was signaling whenever he had rock and the partner was either choosing rock or sending back a signal to indicate that he did not have rock. Both Pair 7 and 4 developed a strategy in which both participants chose paper and used signaling to indicate if any of them had paper blocked. In such situations they were choosing rock by default. Interestingly, Pair 7 started using this strategy only in the second half of the experiment, with no usage of signaling during the first half, at all. Finally, Pair 2 showed a situation in which one of the partners was consistently signaling presence of a specific option (paper), while the other person was experimenting with different communicative strategies, while typically choosing scissors when in doubt. 


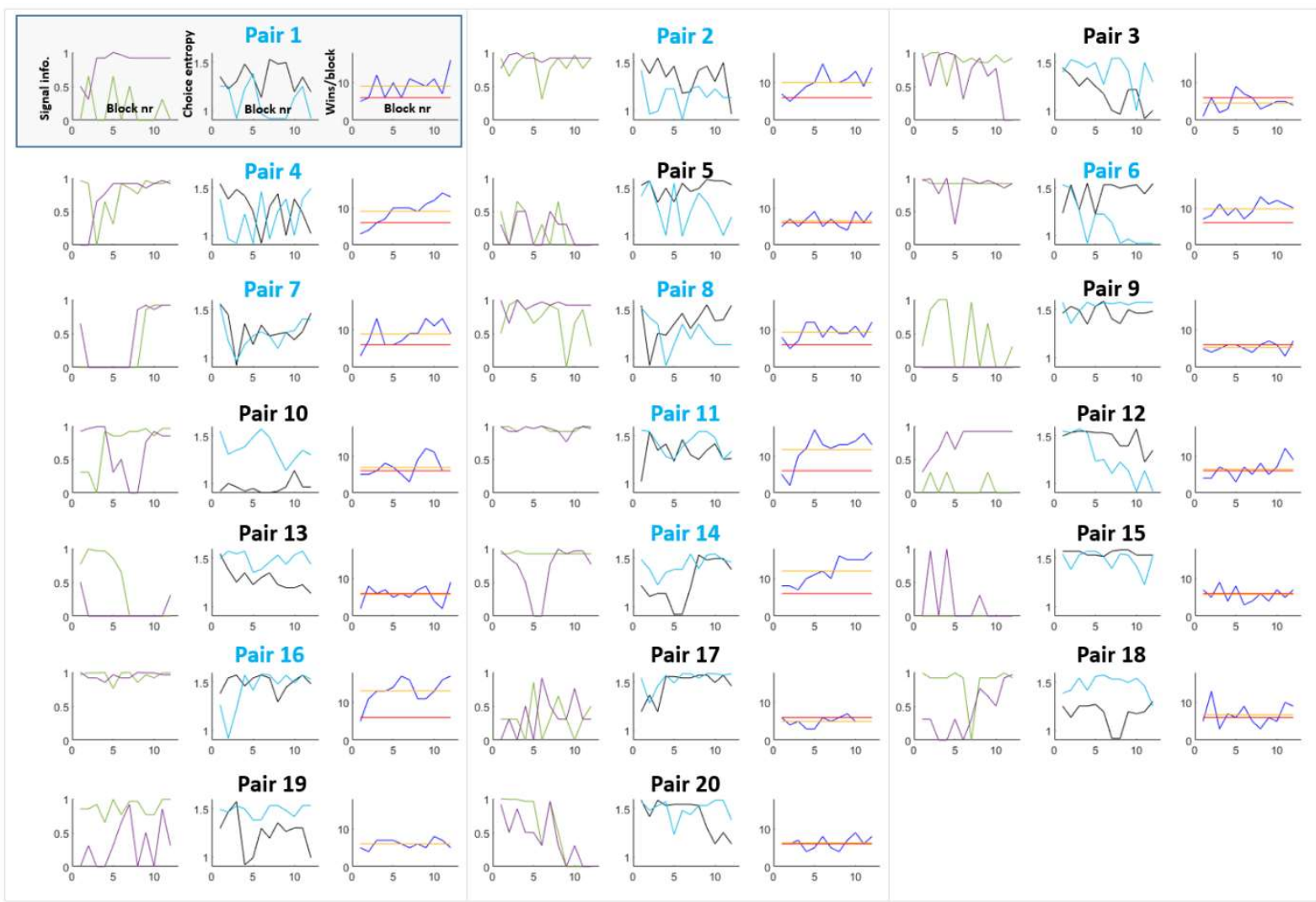

Figure 7. Signal informativeness (graphs with purple and green lines), choice entropy (graphs with black and teal lines) and numbers of wins (graphs with blue, red, and orange lines) across blocks for each of 20 pairs that participated in Experiment 2. In plots with signal informativeness purple and green lines represent individual participant in each pair. In plots with choice entropy black and teal lines represent individual participant in each pair. In plots with average wins blue lines correspond to number of wins by each pair in cooperative trials in each block, while the orange lines reflect their average. The red lines correspond to the number of wins expected by chance if both participants responded randomly (six wins per block). Pairs in blue were classified as successful pairs and pairs in black as unsuccessful pairs.

\subsection{DISCUSSION}

The results of Experiment 2 show that at the group level participants were able to utilize minimal reciprocal communication to improve their performance in the task. Approximately 
half of the tested pairs of participants developed simple communication strategies that allowed them to significantly improve their task performance. At the same time, predictability of participants' choices remained significantly higher in the cooperative than in the competitive context, showing that participants simultaneously used both strategies to facilitate successful cooperation: behavioral predictability and communication. Importantly, signal informativeness negatively correlated with choice entropy. It indicates that participants who were sending more informative signals were also making their behavior more predictable, what translated to on average higher success in the cooperative task. Hence, successful pairs of participants used both possible strategies simultaneously to improve cooperation: behavior predictability and communication.

\section{EXPERIMENT 3}

Experiment demonstrated that pairs of people can use both behavioral predictability and minimal forms of communication to facilitate successful cooperation. In Experiment 3 we set out to further investigate whether people may still use behavioral predictability if they are allowed to freely communicate with each other during the task. We hypothesized that on the one hand, people might always combine communication with behavioral predictability to maximize the probability of successful coordination even if it is associated with high redundancy of coordination strategies. On the other hand, if people dynamically adjust to what extent they use communication and predictability, in order to reduce the required effort, then having the possibility to freely communicate should lead to abandoning of the use of predictability, as verbal communication can easily disambiguate any potential uncertainty.

\subsection{METHODS}

\subsubsection{Participants}


Forty-two volunteers participated in the study comprising 21 dyads. One dyad was excluded from the analysis, because of malfunction of the voice recording device. The mean age of the remaining 40 participants was 25.8 years $(\mathrm{SD}=4.7)$. Twenty-one participants were female. All participants were right-handed, except for three left-handed. The participants represented diverse national and linguistic backgrounds and none of them was a native English speaker. Seven participants did not know the Rock-Paper-Scissors game prior to participation in the study.

\subsubsection{Procedure}

The procedure of Experiment 3 was identical to Experiment 2 with one exception during the cooperative task participants were not allowed to send signals before making their choices (just like in Experiment 1). Instead, they could speak to each other in English while choosing from the options displayed on their screen (and only during this period). Their conversations were recorded and transcribed after the experiment.

\subsubsection{Research design and data analysis}

Just as Experiment 2, Experiment 3 comprised a single-factor repeated-measures design comparing competitive vs. cooperative Task. Experiment 3 used the same dependent variables as Experiment 1, but also included one additional dependent variable: speech entropy.

Speech entropy is a measure of average information (measured in bits) transmitted by each participant through speech within a block of the cooperative task and was calculated using the following formula:

$$
\text { Speech entropy }=-\sum_{i=1}^{n}\left(p_{i} \log _{2} \mathrm{p}_{i}\right)
$$

With $n$ being the number of unique words used by a participant within a given block and $p_{i}$ being the probability that a word $i$ was used by a participant within a given block (calculated as the number of times that a participant used a word $i$ divided by the total number of non-unique 
words that a participant used during a given block). Speech entropy could take the value of 0 bits if a participant did not speak during a block. In cases when participants used spoken language during the task, speech entropy increased with the number of unique words used by a participant and decreased the more the distribution of usage of these words differed from a uniform distribution. For instance, if a participant used 10 unique words during a block, but $90 \%$ of the time she used only three of these words, then speech entropy would be only slightly higher than if she used only 3 words throughout this block (i.e. slightly higher than 1.6 bit), and much lower than if she used all 10 words equally often (i.e. 3.3 bit).

There were two reasons why we decided to use speech entropy as a quantitative measure of the amount of verbal signaling used by the participants. First, in comparison to the number of unique words, speech entropy allowed us to account for situations where only a few words are instrumental to communication, while other, much less frequent words, are used only as embellishments, or mistakes. Second, because speech entropy was measured in bits, it became possible to compare it with choice entropy, which was also measured in bits.

Preregistration for Experiment 3 is available at: https://aspredicted.org/2j84j.pdf Full results are available in the supplementary materials S2.

\subsection{RESULTS}

In the competitive task $2.2 \%(S D=1.7 \%)$ trials were excluded due to participants' mistakes (pressing a "blocked" option, pressing the same button twice etc.). This amounted to an average of 4.65 mistakes per participant (the range in both tasks was between 0 and 13 mistakes). In the cooperative task $2.3 \%(S D=3.9 \%)$ trials were excluded due to participants' mistakes. This amounted to on average 5 mistakes per participant (the range in both tasks was between 0 and 39 mistakes). The difference in the number of mistakes was not significantly higher for the cooperative than for the competitive task $(\mathrm{t}(39)=-0.34, \mathrm{p}=0.73)$. 
Participants showed a stronger preference to choose rock (37.1\%) than scissors $(32.2 \%)$, and scissors more than paper $(29.8 \%)$ in the competitive task $(F(2,78)=6.70, p=0.002$, partial $\left.\eta^{2}=0.15, \mathrm{BF}_{10}=246\right)$, and preferred rock $(35.3 \%)$ over paper $(32.7 \%)$ and paper over scissors $(31.1 \%)$ in the cooperative task $\left(F(2,78)=8.76, p<0.001\right.$, partial $\left.\eta^{2}=0.18, \mathrm{BF}_{10}=2444\right)$.

\subsubsection{Win rate}

A Wilcoxon's test revealed that participants obtained significantly more points in the cooperative than in the competitive task $(Z(39)=0, \mathrm{P}<0.001$, rank biserial correlation $=0$, $\left.\mathrm{BF}_{10}=1.49 \mathrm{e}+27\right)$. We also investigated the number of wins at the level of individual blocks with an additional two-way repeated-measures ANOVA $(2 \times 12)$ with the factors Task (competitive vs. cooperative) and Block (block 1 to block 12). The results revealed a significant main effect of Task $\left(F(1,39)=1149.16, p<0.001\right.$, partial $\left.\eta^{2}=0.97, \mathrm{BF}_{\text {inclusion }}=\mathrm{inf}\right)$, a significant main effect of block $\left(F(11,429)=5.64, \quad p<0.001 \quad\right.$ Greenhouse-Geisser corrected, partial $\eta^{2}=0.13$, $\left.\mathrm{BF}_{\text {inclusion }}=6.3 \mathrm{e} 7\right)$, and a significant interaction $(F(11,429)=7.01, p<0.001$ Greenhouse-Geisser corrected, partial $\left.\eta^{2}=0.15, \mathrm{BF}_{\text {inclusion }}=7.1 \mathrm{e} 5\right)$. 

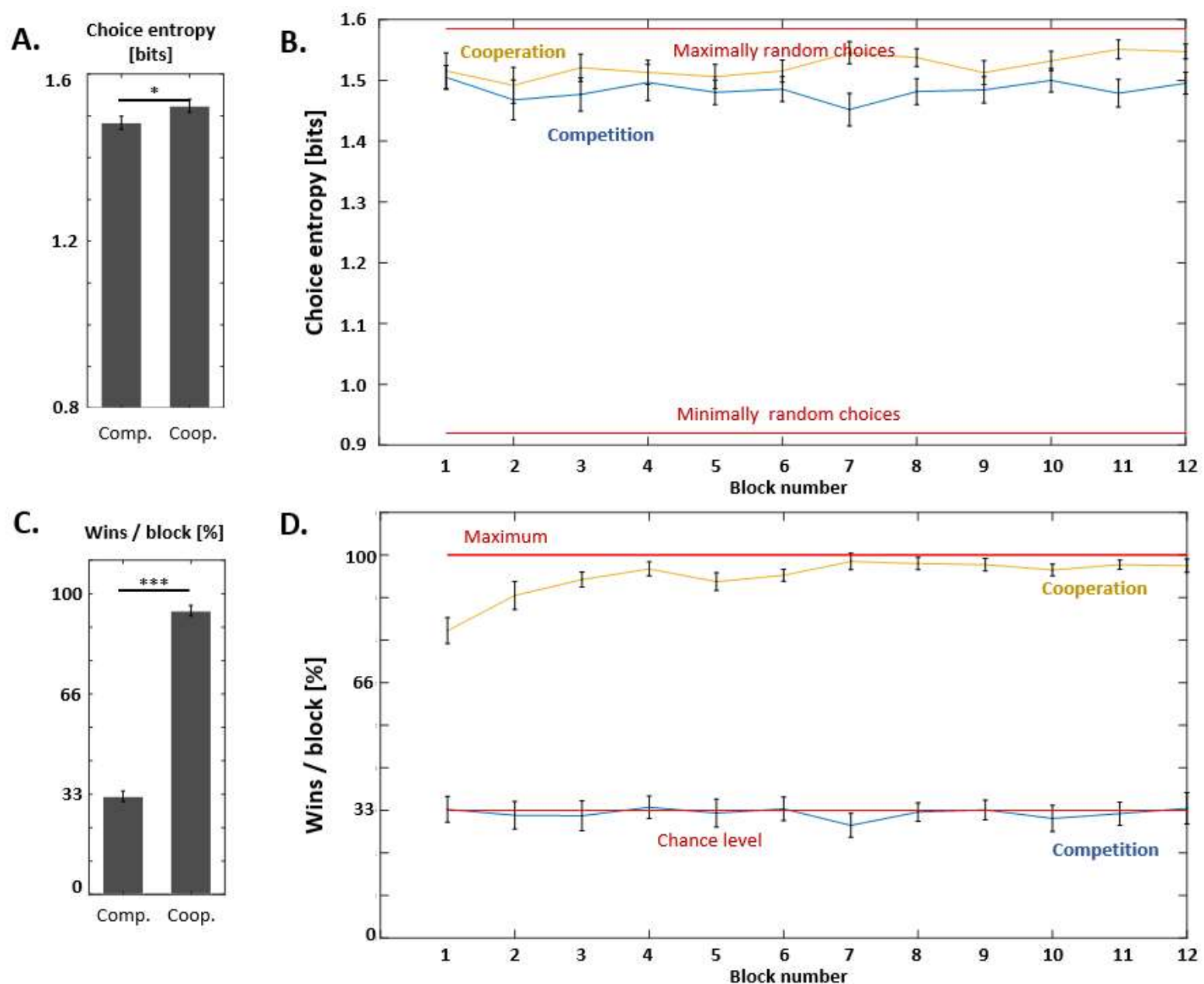

Fig 8. Results of Experiment 3. Bar graphs on the left illustrate the differences between competitive and cooperative task in average choice entropy (A) and win rate (C). Line graphs on the right show average choice entropy (B) and win rate (D) across individual blocks in competitive and cooperative task. Brackets indicate 95\% CIs.

\subsubsection{Choice entropy}

A Wilcoxon's test revealed that choice entropy was significantly higher in the cooperative than in the competitive task $(Z(39)=181, \mathrm{p}=0.002$, rank biserial correlation $=0.56$, $\left.\mathrm{BF}_{10}=2.49\right)$.

In the next step, we investigated choice entropy at the level of individual blocks. We conducted an additional two-way repeated-measures ANOVA $(2 \times 12)$ with the factors Task 
(competitive vs. cooperative) and Block (from block 1 to block 12). The results revealed that the main effect of Task $\left(F(1,57)=6.12, p<0.001\right.$, partial $\left.\eta^{2}=0.14, B_{\text {inclusion }}=5.7 \mathrm{e} 8\right)$ was significant. The main effect of Block was not significant $(F(11,429)=1.86, p=0.084$ Greenhouse-Geisser corrected, partial $\left.\eta^{2}=0.05, \mathrm{BF}_{\text {inclusion }}=0.003\right)$, but the interaction effect was significant, although the Bayesian analysis strongly favored its exclusion from the model $\left(F(11,627)=2.15, p=0.042\right.$ Greenhouse-Geisser corrected, partial $\left.\eta^{2}=0.05, B F_{\text {inclusion }}=7.6 \mathrm{e}-4\right)$.

\subsubsection{Correlations between variables}

As in Experiment 2 and in contrast to Experiment 1 in the competitive task we did not find a significant correlation between choice entropy and win rate $(\operatorname{rho}(38)=-0.08, p=0.64)$. In the cooperative task this relationship was once again significant $(\operatorname{rho}(38)=0.57, p<0.001)$. In contrast to the previous experiments it was positive, rather than negative, meaning that better performance was associated with more random, rather than less random choices. It is likely that pairs that needed more time to agree on linguistic conventions used to efficiently solve the task were relying for longer on a less efficient strategy of making predictable choices. In the cooperative task we also investigated the relationship of speech entropy with win rate $(\operatorname{rho}(38)=0.01, p=0.95)$ and choice entropy $(\operatorname{rho}(38)=0.21, p=0.19)$ but neither of them was significant.

\subsubsection{Cooperation strategies}

In contrast to Experiment 2, in Experiment 3 participants were allowed to speak with each other before making a choice. This resulted in nearly perfect performance in all but one pair of participants, which however still achieved above chance-level performance (Pair 15, average win rate across all blocks 51\%). Another pair (Pair 7) performed the first half of the cooperative task in a sub-efficient manner, with only one participant communicating his choice to the partner (leading to 12.6 wins, or $70.4 \%$ win rate, in the first 6 blocks), but after a discussion during the sixth block, the pair agreed to change their strategy, leading to 
improvement in their performance to $99.1 \%$ win rate in the last six blocks. All remaining pairs adopted some form of a strategy that allowed near perfect performance during the first 2-3 blocks. The most common strategy was to have one participant propose which of the three options to choose, and then have the other person to approve or disapprove the choice (by saying “yes"/“ok", or "no"/“blocked"). In case of disapproval the first person could give a second proposal, which this time was guaranteed to be correct, or in some cases the second participant could present a proposal, although with a risk that it might be blocked. This was by far the most common strategy used by 12 pairs. Three further pairs also used the strategy of proposing a common choice, but instead of using words reflecting approval or disapproval, they only used the words "rock", "paper" and "scissors". In this case repeating the partner's choice meant approval, while proposing a different choice meant disapproval. Two pairs used a strategy in which one person begins by saying which two options she or he has available (e.g. rock and paper), and then the second person makes the choice (e.g. paper). Finally, the last two pairs adopted a strategy in which the first person tells which option is blocked, and then the second person makes a choice which option they should choose.

\subsubsection{Information content in verbal communication}

All pairs engaged in some form of verbal communication. To estimate the quantitative characteristics and evolution across time of verbal communication between participants we performed analyses on the transcripts of words that the participants used during the task. We calculated the number of unique words used by each participant in each block, as well as entropy of distribution of words used by each participant in each block. Speech entropy was calculated by treating speech in a similar way to choices in the rock-paper-scissors game: each spoken word was treated as an instance of sending one type of signal, and the distribution of the number of times that each word was used within a block served as data for calculations. Speech entropy then becomes a measure of the number of bits of information that got transmitted on average in 
each trial within a given block. The difference between quantifying choice entropy and speech entropy in this case is that while choices were limited to a space of only three possible options, speech was in principle unlimited.

We observed that at the group-level both the number of unique words and speech entropy were highest in the first block and then gradually decreased and stabilized from the seventh block onwards. Interestingly, the average speech entropy for the whole group stabilized at the same level as choice entropy, which was also at the level of choice entropy for maximally random choices.

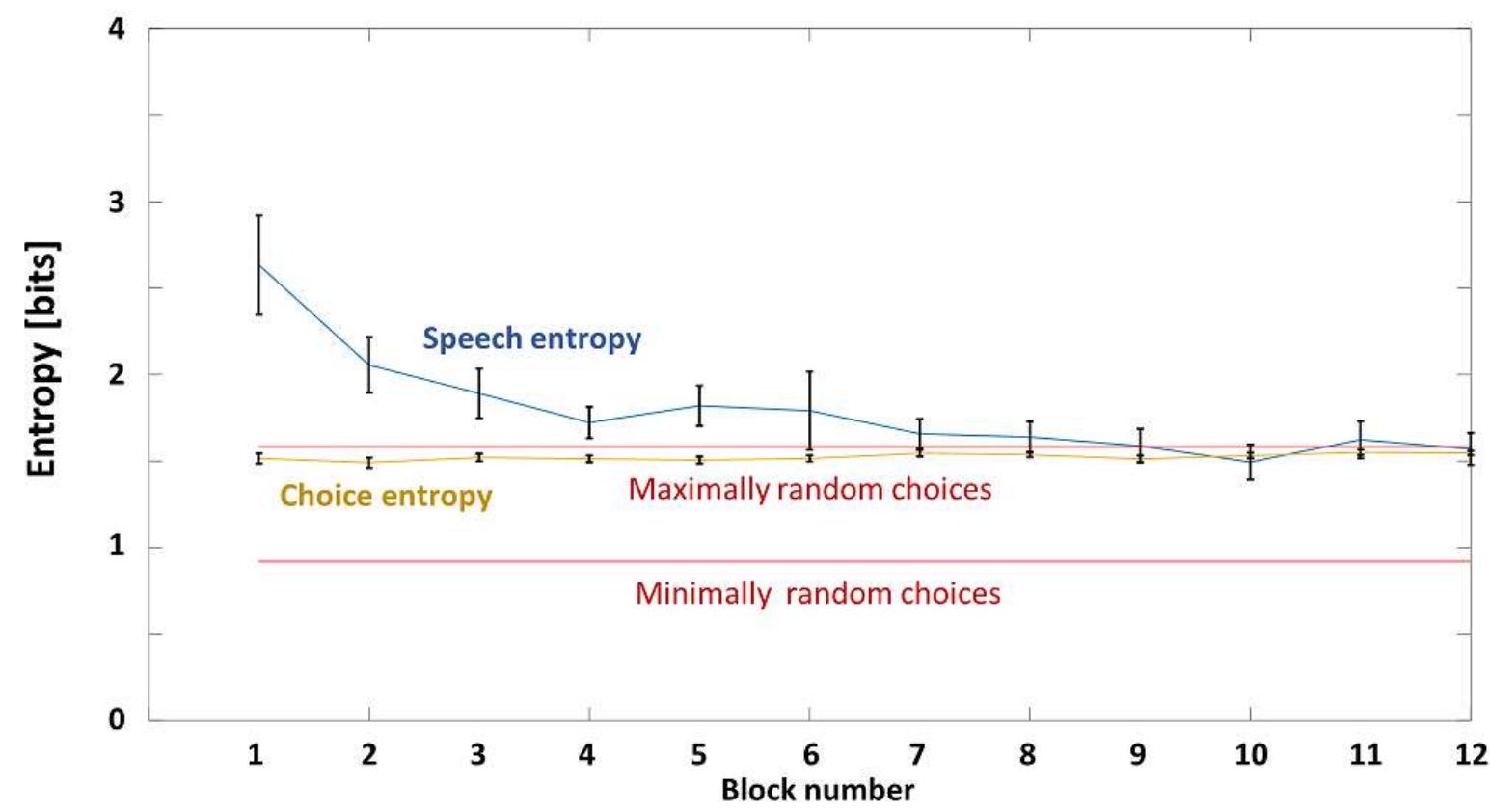

Fig 9. Comparison of average choice entropy and speech entropy across blocks in the cooperative task in Experiment 3. The average speech entropy was greatest in the first block and then gradually decreased until it converged with the entropy of choices, which reflected the amount of information that needed to be conveyed through verbal communication to succeed in the task, illustrating optimization of verbal communication across time. Speech entropy converged to the level of maximally random choices, because in Experiment 3 entropy of participants' choices was at that level. Brackets indicate 95\% CIs. 


\subsection{DISCUSSION}

Experiment 3 has shown that when unlimited verbal communication was available all participants were able to use it to improve task performance, and all but one pair of participants used it to achieve ceiling-level performance. At the same time, the behavioral choices of participants became fully unpredictable, and reached the level of choice entropy that was even higher than in the competitive context. This illustrates that behaving predictably and communicating are two interchangeable strategies of facilitating successful cooperation, and that if one of them allows to fully maximize cooperation success then there is no need to use the other anymore.

Finally, we found that the information content of verbal communication (measured by speech entropy) between participants in the cooperative context sharply decreased over time until it reached the level comparable to uncertainty of participants' choices that it needs to explain. This shows that participants optimized their verbal communication to make it not only fully informative, but also to decrease redundancy and effort needed to coordinate.

\section{GENERAL DISCUSSION}

We conducted three experiments in which participants played a modified version of the rock-paper-scissors game, either cooperating or competing with one another. Across experiments we manipulated the degree to which participants could communicate with each other to facilitate successful cooperation. In Experiment 1 no communication was possible, in Experiment 2 participants were allowed minimal reciprocal communication, and in Experiment 3 unlimited reciprocal communication using language was allowed.

Our goal was to answer three research questions. First, we asked whether people are able to use behavioral predictability to facilitate the coordination required for joint decision 
making. The results of Experiment 1, in which no communication between participants was possible, provide evidence that the answer to this question is yes. Approximately half of the pairs in our task were able to achieve above-chance-level performance through making their choices more predictable in the cooperative task, making successful coordination more likely. This finding is in line with previous studies reporting similar results (Schelling, 1960; Sugden, 1995; Vesper et al., 2011).

The second question was whether people are able to use behavioral predictability and communication, at the same time, to facilitate coordination. The results of Experiment 2 confirmed this. In a situation in which pairs of participants could use a minimal (one-bit) form of reciprocal communication, they kept ensuring behavioral predictability to succeed in the cooperative task. While not all pairs did so successfully, some achieved a level of performance, that was much higher than that of the best pairs from Experiment 1. This additional benefit was due to establishing simple communicative conventions in the course of the interaction and was observed in approximately half of the pairs.

Finally, the third question was whether people still use behavioral predictability if they are allowed to use the full power of conventional verbal communication. In Experiment 3, when participants were allowed to speak with each other (unlimited reciprocal communication), we found that almost all pairs were able to rapidly reach ceiling-level performance in the cooperative task. At the same time their choices in the task became fully random. This shows that when language was efficient enough to fully disambiguate participants' choices, participants did not need to structure their responses anymore. In fact, participants' individual choices were less predictable when cooperating than when competing. This implies that competitive context does not encourage fully random responses, as would be predicted by the rational agents model (Nash, 1950; Osborne \& Rubinstein, 1994; Taylor \& Jonker, 1978), but 
involves a certain degree of non-randomness (for similar results see: (Wang, Xu, \& Zhou, 2014; Xu, Zhou, \& Wang, 2013)).

When contrasting communication and behavioral predictability our study found that communication was a much more powerful method of facilitating successful cooperation. In Experiment 1 where participants could use only behavioral predictability they achieved performance only slightly above the chance level, while in Experiment 3, which allowed unlimited communication, all pairs except for one achieved ceiling-level performance. However, this finding should not be generalized too quickly to other joint decision contexts. First, the design of our task made it in principle impossible to consistently achieve perfect performance in Experiment 1 whereas in Experiment 2 and 3 this option was at least theoretically within reach. Future studies could use tasks in which both strategies are equally feasible and investigate to what extent people prefer to use one over another, and what factors might influence this preference. Second, our study involved cooperation to achieve a joint decision. In tasks that require coordination of bodily actions, such as dancing or cooperative sports, the relative influence of behavioral predictability and communication might yield an opposite pattern of results, with the former playing much larger role.

In our study, we found that when participants could not use communication (Experiment 1) they often tried to ensure predictability of their behavior by repeatedly choosing the same option, hoping that their partner will do the same. However, how did they decide which option to choose in the first place? One possibility is that the participants thought of one of the options as a focal point (Schelling, 1960; Sugden, 1995), i.e. an option that is more salient than the others. In the case of our task, the most salient option might have been "rock", as it is the first symbol that is present in the English name of the game ("Rock-Paper-Scissors") on which our study was based. Indeed, we found that participants were more likely to choose "rock" than the other options in the cooperative task in Experiments 1 and 3, while in Experiment 2 participants 
did not show preference for any option. However, in Experiment 3 participants were similarly likely to choose "rock" both in the cooperative and in competitive context, suggesting that they had an overall preference to choose this option, rather than the preference to use it in a strategic way as a focal point. In Experiment 1 participants had a significant preference to choose rock over the other options in both tasks, but this preference was stronger in the cooperative $(42.2 \%)$ than in the competitive task (36.7\%). This difference provides some indication that participants might have used a focal point strategy. However, none of the participants mentioned that they tried to use rock more frequently in a post-experimental debriefing session. Thus, it is possible that focal points may help with coordinating decisions due to shared implicit preferences (Schelling, 1960), in a linguistic context see also: (Tylén, Fusaroli, Bundgaard, \& Østergaard, 2013)).

In Experiment 1 participants could not communicate with each other and in Experiment 3 they could use conventional language to communicate. In contrast, Experiment 2 presented them with an intermediate situation in which they could engage in a minimal form of reciprocal communication. In order to use communication effectively they had to develop basic communication conventions from scratch. As shown by the comparison of successful and unsuccessful pairs from Experiment 2, only about half of the tested pairs were able to bootstrap a functioning communication system. This is in line with previous studies on development of new communicative conventions, which also found that communication success in such circumstances is highly variable (Galantucci, 2005; Galantucci \& Garrod, 2010; T. C. ScottPhillips \& Kirby, 2010). Those pairs that did succeed in our task, developed various signaling conventions. In most cases signaling was used to indicate presence or absence of specific options, for example: sending a signal was indicating that one has or does not have "scissors". Most of the successful pairs in our study developed some form of bidirectional communication, although we found pairs in which communication was predominantly unidirectional, i.e. only 
one of the participants was sending informative signals (Pair 1). Finally, as illustrated by the example of Pair 7, a successful communication convention can emerge spontaneously in the middle of the experiment as a novel invention rather than in a process of gradual mutual readjustment.

We observed evolution of communicative behavior not only in Experiment 2, where participants developed a novel (minimal) communication convention, but also in Experiment 3, where participants could use full conventional linguistic communication. In Experiment 3 we observed that the entropy of spoken communication for each participant was highest in the first block and then gradually decreased across the subsequent blocks until it reached the level of entropy representing the amount of uncertainty present in the task that participants had to disambiguate. Thus, participants were simplifying their verbal communication across the time course of the experiment until it reached the level of maximal simplicity that was needed to succeed in the task. This finding is in line with previous studies showing that across time people optimize their verbal communication to make it more efficient for succeeding in a joint task (e.g. (Clark \& Wilkes-Gibbs, 1986; Fusaroli et al., 2012; Lelonkiewicz \& Gambi, 2020; Schober \& Clark, 1989)). Most likely this is a specific manifestation of a more general tendency to reduce complexity or the level of required effort (Chater \& Vitányi, 2003; Zipf, 1949) that includes optimization of linguistic communication (Mahowald, Fedorenko, Piantadosi, \& Gibson, 2013; Piantadosi, Tily, \& Gibson, 2011).

\section{CONCLUSIONS}

Across three experiments we manipulated the degree to which participants could use communication to successfully achieve a joint decision. We found that behavioral predictability and communication are two complementary strategies that can facilitate interpersonal coordination and that availability of communication reduced constraints on behavioral 
predictability in joint action coordination. If the bandwidth of the available communication channel is limited people are able to combine both strategies and to adaptively switch between them. Even when the opportunities for communication are minimal, people are capable of bootstrapping new communication systems on the fly and to use them for cooperation.

Our study introduces a novel approach to studying cooperative social behavior which puts emphasis on comparing different strategies towards achieving successful coordination. Further studies of this kind can improve our understanding of what are the factors that make people communicate (or not) when coordinating their actions and decisions during cooperative activities.

\section{Acknowledgments}

This study was funded by the European Research Council under the European Union's Seventh Framework Programme (FP7/2007-2013) / ERC grant agreement $n^{\circ}$ 609819, Constructing Social Minds: Coordination, Communication, and Cultural Transmission (SOMICS).

\section{References}

Chater, N., \& Vitányi, P. (2003). Simplicity: A unifying principle in cognitive science? Trends in Cognitive Sciences, 7(1), 19-22.

Clark, H. H., \& Wilkes-Gibbs, D. (1986). Referring as a collaborative process. Cognition, 22(1), 1-39.

Crawford, V. P., \& Haller, H. (1990). Learning how to cooperate: Optimal play in repeated coordination games. Econometrica: Journal of the Econometric Society, 571-595.

Fay, N., Ellison, M., \& Garrod, S. (2014). Iconicity: From sign to system in human communication and language. Pragmatics \& Cognition, 22(2), 244-263.

Fusaroli, R., Bahrami, B., Olsen, K., Roepstorff, A., Rees, G., Frith, C., \& Tylén, K. (2012). Coming to terms: Quantifying the benefits of linguistic coordination. Psychological science, 23(8), 931939.

Galantucci, B. (2005). An experimental study of the emergence of human communication systems. Cognitive science, 29(5), 737-767.

Galantucci, B. (2017). Experimental semiotics. In Oxford research encyclopedia of linguistics.

Galantucci, B., \& Garrod, S. (2010). Experimental semiotics: A new approach for studying the emergence and the evolution of human communication. Interaction Studies: Social Behaviour and Communication in Biological and Artificial Systems. 
Galantucci, B., \& Garrod, S. (2011). Experimental semiotics: a review. Frontiers in human neuroscience, $5,11$.

Glover, S., \& Dixon, P. (2017). The role of predictability in cooperative and competitive joint action. Journal of Experimental Psychology: Human Perception and Performance, 43(4), 644.

Lelonkiewicz, J. R., \& Gambi, C. (2020). Making oneself predictable in linguistic interactions. Acta psychologica, 209, 103125.

Lindsay, L., Gambi, C., \& Rabagliati, H. (2019). Preschoolers optimize the timing of their conversational turns through flexible coordination of language comprehension and production. Psychological science, 30(4), 504-515.

Mahowald, K., Fedorenko, E., Piantadosi, S. T., \& Gibson, E. (2013). Info/information theory: Speakers choose shorter words in predictive contexts. Cognition, 126(2), 313-318.

McEllin, L., Knoblich, G., \& Sebanz, N. (2018). Distinct kinematic markers of demonstration and joint action coordination? Evidence from virtual xylophone playing. Journal of Experimental Psychology: Human Perception and Performance, 44(6), 885.

Mehta, J., Starmer, C., \& Sugden, R. (1994). Focal points in pure coordination games: An experimental investigation. Theory and Decision, 36(2), 163-185.

Michael, J., McEllin, L., \& Felber, A. (2020). Prosocial effects of coordination-What, how and why? Acta psychologica, 207, 103083.

Michael, J., \& Pacherie, E. (2015). On commitments and other uncertainty reduction tools in joint action. Journal of Social Ontology, 1(1), 89-120.

Misyak, J., Noguchi, T., \& Chater, N. (2016). Instantaneous conventions: The emergence of flexible communicative signals. Psychological science, 27(12), 1550-1561.

Nash, J. F. (1950). Equilibrium points in n-person games. Proceedings of the National Academy of Sciences, 36(1), 48-49.

Osborne, M. J., \& Rubinstein, A. (1994). A course in game theory: MIT press.

Pezzulo, G., Donnarumma, F., Dindo, H., D'Ausilio, A., Konvalinka, I., \& Castelfranchi, C. (2019). The body talks: Sensorimotor communication and its brain and kinematic signatures. Physics of life reviews, 28, 1-21.

Piantadosi, S. T., Tily, H., \& Gibson, E. (2011). Word lengths are optimized for efficient communication. Proceedings of the National Academy of Sciences, 108(9), 3526-3529.

Raihani, N. (2021). The Social Instinct: How Cooperation Shaped the World. London: Jonathan Cape.

Rand, D. G., \& Nowak, M. A. (2013). Human cooperation. Trends in Cognitive Sciences, 17(8), 413425.

Sabu, S., Curioni, A., Vesper, C., Sebanz, N., \& Knoblich, G. (2020). How does a partner's motor variability affect joint action? PLoS One, 15(10), e0241417.

Sacheli, L. M., Tidoni, E., Pavone, E. F., Aglioti, S. M., \& Candidi, M. (2013). Kinematics fingerprints of leader and follower role-taking during cooperative joint actions. Experimental Brain Research, 226(4), 473-486.

Sachs, J. L., Mueller, U. G., Wilcox, T. P., \& Bull, J. J. (2004). The evolution of cooperation. The Quarterly Review of Biology, 79(2), 135-160.

Schelling, T. C. (1960). The strategy of conflict: Harvard university press.

Schober, M. F., \& Clark, H. H. (1989). Understanding by addressees and overhearers. Cognitive psychology, 21(2), 211-232.

Scott-Phillips, T. (2014). Speaking our minds: Why human communication is different, and how language evolved to make it special: Macmillan International Higher Education.

Scott-Phillips, T. C., \& Kirby, S. (2010). Language evolution in the laboratory. Trends in Cognitive Sciences, 14(9), 411-417.

Sugden, R. (1995). A theory of focal points. The Economic Journal, 105(430), 533-550.

Tamariz, M. (2017). Experimental studies on the cultural evolution of language. Annual Review of Linguistics, 3, 389-407.

Taylor, P. D., \& Jonker, L. B. (1978). Evolutionary stable strategies and game dynamics. Mathematical biosciences, 40(1-2), 145-156. 
Tylén, K., Fusaroli, R., Bundgaard, P. F., \& Østergaard, S. (2013). Making sense together: A dynamical account of linguistic meaning-making. Semiotica, 2013(194), 39-62.

Tylén, K., Weed, E., Wallentin, M., Roepstorff, A., \& Frith, C. D. (2010). Language as a tool for interacting minds. Mind \& Language, 25(1), 3-29.

Vesper, C., Schmitz, L., \& Knoblich, G. (2017). Modulating action duration to establish nonconventional communication. Journal of Experimental Psychology: General, 146(12), 1722.

Vesper, C., Schmitz, L., Safra, L., Sebanz, N., \& Knoblich, G. (2016). The role of shared visual information for joint action coordination. Cognition, 153, 118-123.

Vesper, C., van der Wel, R. P., Knoblich, G., \& Sebanz, N. (2011). Making oneself predictable: Reduced temporal variability facilitates joint action coordination. Experimental Brain Research, 211(34), 517-530.

Wang, Z., Xu, B., \& Zhou, H.-J. (2014). Social cycling and conditional responses in the Rock-PaperScissors game. Scientific Reports, 4, 5830.

Xu, B., Zhou, H.-J., \& Wang, Z. (2013). Cycle frequency in standard rock-paper-scissors games: evidence from experimental economics. Physica A: Statistical Mechanics and its Applications, 392(20), 4997-5005.

Zipf, G. K. (1949). Human behavior and the principle of least effort: an introd. to human ecology. 